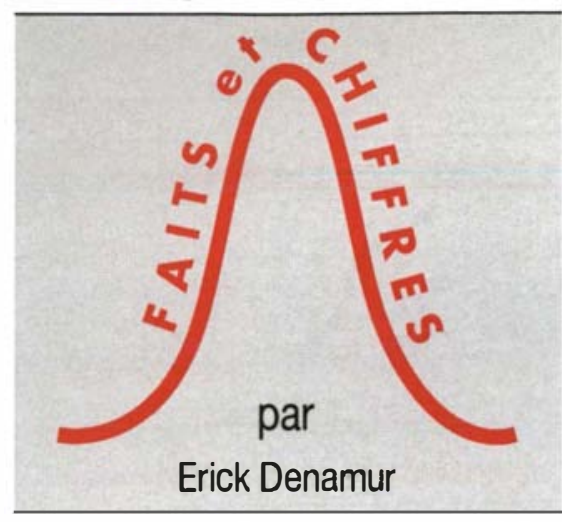

médecine/sciences $1994 ; 10: 1157-60$

\title{
Les généticiens sur la piste des premiers Américains
}
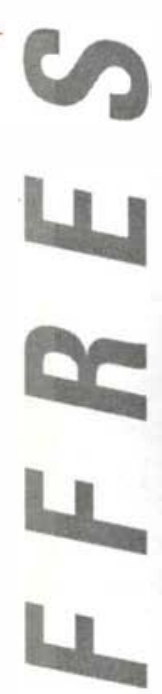

$\mathbf{0}$ ui étaient les premiers Américains ? Quand et comment ont-ils peuplé le continent américain ? Voici des questions dont les réponses sont loin de faire l'unanimité, que ce soit parmi les archéologues, les anthropologues, les linguistes ou les généticiens. Tout commence à Clovis, dans le Nouveau Mexique, en 1932, quand des archéologues découvrent des pierres taillées ayant servi d'outils et des restes de campements de chasseurs datés de 11000 ans avant maintenant (AM). L'hypothèse, fondée sur ces découvertes, faisait des hommes de Clovis des chasseurs venus d'Asie passés sur un pont de terre de la Sibérie vers l'Alaska assez peu de temps avant cette date $(\approx 12000$ ans AM).

\section{L'hypothèse Greenberg}

En 1986, Joseph Greenberg, linguiste à l'université de Stanford (Californie) proposa avec Christy Turner et Stephen Zegura, deux anthropologues de l'université d'Arizona, une théorie sur le peuplement du continent américain fondée sur des données linguistiques, dentaires et génétiques : tous les ancêtres des Indiens américains seraient arrivés par trois vagues successives [1]. La première migration, correspondant aux Amérindiens, serait survenue aux alentours de 12000 ans AM et aurait donné la culture de Clovis. Puis, plus récemment, seraient venus en migrations séparées les NaDene sur la côte Nord-Ouest de l'Amérique du Nord et les Aleut-Eskimo dans l'extrême
Nord du continent. Chaque vague migratoire aurait apporté son propre langage ancestral qui aurait donné ensuite de nombreux descendants : l'Aleut-Eskimo, le NaDene (parlé par les populations de la côte NordOuest) et l'Amérindien parlé par le reste des Indiens du Nord et par les Indiens du Sud de l'Amérique. Turner a identifié, d'une part, un ensemble de caractéristiques de la dentition qui lient les populations du Nord-Est de l'Asie aux Indiens américains préhistoriques et modernes et, d'autre part, des caractéristiques propres aux trois groupes précédemment cités. Enfin, les données génétiques, fondées essentiellement sur l'étude des polymorphismes protéiques de Zagura différenciant ces trois groupes, ont été confirmées par celles de Cavalli-Sforza et al. [2]. Mais cette séduisante hypothèse a été largement remise en cause en 1990 à la conférence de Boulder (Colorado) sur la classification Greenberg [3]. Tout d'abord, la contestation est venue des linguistes eux-mêmes, qui refusent quasi unanimement le concept des groupes avancé par Greenberg. La technique de Greenberg appelée " comparaison de masse " est fondée sur la comparaison de certains mots comme ceux des parties du corps, des pronoms personnels, qui changent peu et sont rarement empruntés à un autre langage. C'est avec des listes de tels mots que Greenberg a comparé des centaines de langages à la recherche de similitudes. Par exemple, il a remarqué que parmi les langages amérindiens

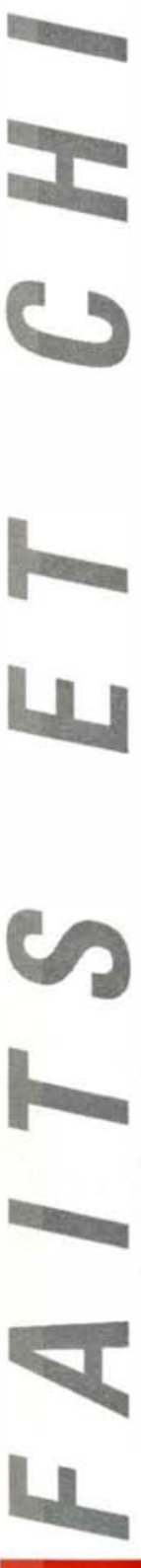

E. Denamur : Inserm U. 120, Hôpital Robert-Debré, 48, boulevard Sérurier. $\mathrm{m} / \mathrm{s} n^{\circ} 11$, vol. 10, novembre 94

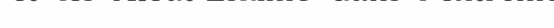


de la Colombie Britannique au Chili, le « $n$ " et le « $m$ » étaient constamment utilisés dans les pronoms singuliers des première et deuxième personnes. Ses adversaires pensent que cette approche n'est pas valide et qu'il faut utiliser une approche plus fine en travaillant sur de petits groupes de langages et comparer non pas des mots, mais des sons. Les groupes Eskimo et NaDene sont ainsi bien retrouvés par les détracteurs de Greenberg mais pour eux l'affirmation d'une famille amérindienne est prématurée [3].

\section{Une culture pré-Clovis}

Ensuite, les archéologues ont apporté des arguments en faveur d'une culture pré-Clovis dans le Nouveau Monde qui pourrait correspondre à une première migration aussi ancienne que 40000 années AM. En effet, cinq sites potentiels témoignant de la présence de l'homme entre 33000 et 13000 années AM ont été mis en évidence sur l'ensemble du continent (Canada, Pennsylvanie - USA, Venezuela, Brésil et Chili). Cette culture pré-Clovis serait fondée sur l'utilisation du bois et le ramassage des plantes plutôt que sur les outils de pierre et la chasse. Toutefois, là encore, la véracité de ces sites ne fait pas le consensus [4].

\section{L'ADN mitochondrial}

Plusieurs groupes de généticiens ont alors tenté d'apporter des éléments au problème en étudiant l'ADN mitochondrial (ADNmt). En effet, l'ADNmt a été utilisé pour étudier les origines et les migrations des populations humaines [5]. L'avantage de l'ADNmt sur l'ADN nucléaire est qu'il accumule les changements de bases à une vitesse cinq à dix fois plus rapide que ne le fait l'ADN nucléaire en copie unique, ce qui en fait un outil parfait pour l'étude des différences d'ADN entre les populations humaines. De plus, le fait qu'il soit hérité seulement de la mère et l'absence de recombinaison permettent la détermination d'un arbre phylogénétique sans les ambiguïtés

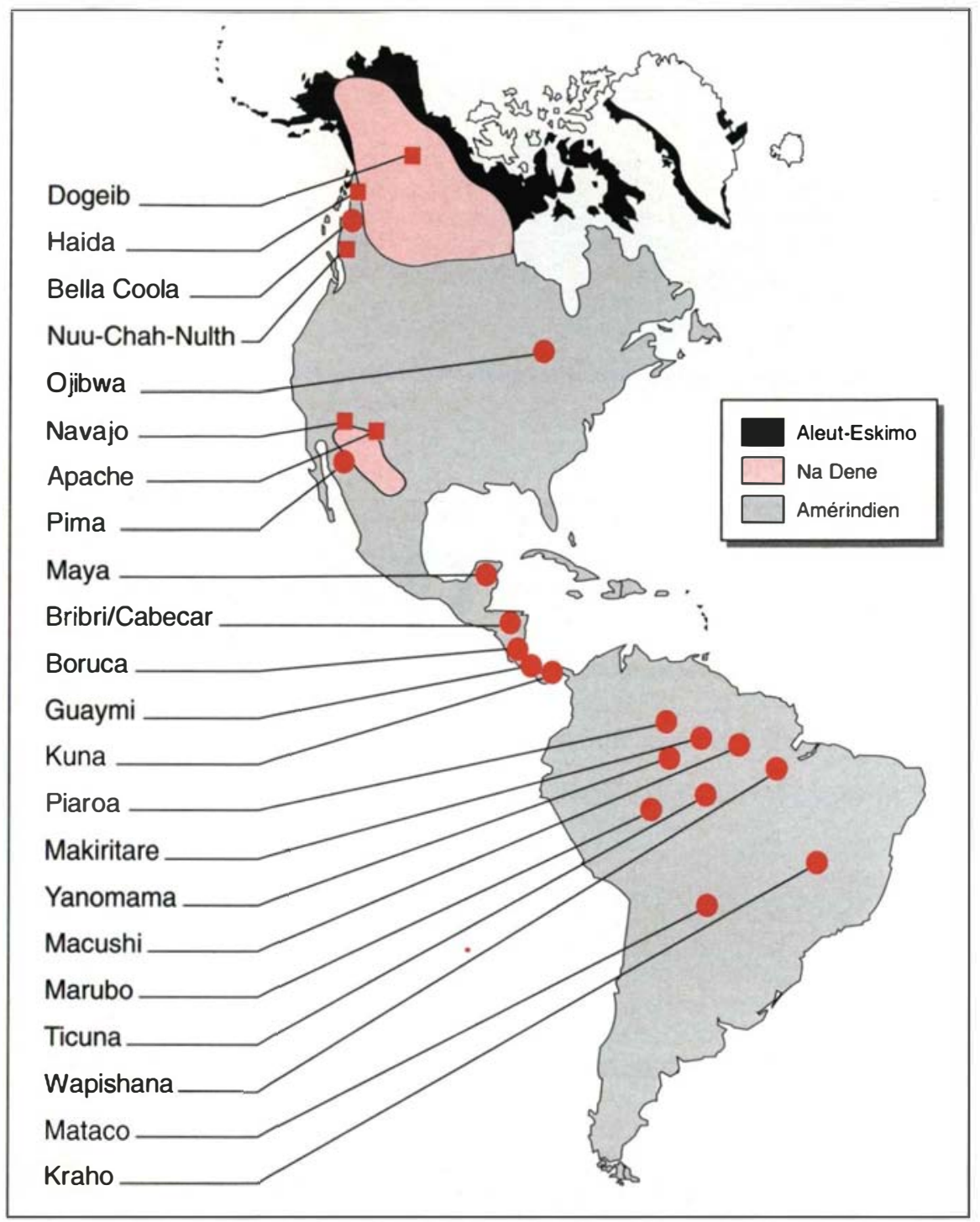

Figure 1. Localisation géographique des populations américaines natives étudiées par Torroni et al. [6]. Les divisions linguistiques (Aleut-Eskimo, NaDene et Amérindien) sont celles proposées par Greenberg et al. [1]. Les cercles et les carrés indiquent respectivement les tribus Amérindiennes et NaDene.

sentes au niveau des gènes nucléaires. En 1993, le groupe de Douglas Wallace (Atlanta, GA, USA), auquel appartient le généticien Antonio Torroni, a étudié la variation de l'ADNmt chez trois cent vingt et un individus provenant de dix-sept populations américaines natives (les Américains natifs sont les Américains présents avant l'arrivée de Christophe Colomb) par ana- lyse de la carte de restriction et par séquençage nucléotidique de l'anse D de produits d'amplification génique in vitro (PCR) [6]. Les résultats ont été comparés à ceux de cinq autres tribus d'Américains natifs ainsi qu'à ceux de populations asiatiques (figure 1). Cette analyse a permis de définir quatre groupes d'haplotypes : haplogroupe A : présence d'un site HaellI en 663 ; 
haplogroupe B : délétion d'une répétition de 9 paires de bases dans une petite région non codante comprise entre les gènes de la cytochrome oxydase II et de l'ARN de transfert lysine, présence du site HaeIII en 16517 ; haplogroupe $C$ : présence des sites AluI en 13262 et 10397 et du site DdeI en 10394 ; haplogroupe D : absence du site AluI en 5176 et présence des sites DdeI en 10394 et AluI en 10397. Les quatre groupes d'haplotypes étaient présents chez les Amérindiens alors que seul l'haplogroupe A était présent chez les NaDene confirmant ainsi la migration séparée des Amérindiens et des NaDene. Chaque haplogroupe semble avoir été formé à partir d'un seul haplotype ancestral. Il y aurait donc eu un goulot d'étranglement génétique lors du passage de l'Asie vers l'Amérique. I a majorité de la variation à l'intérieur des haplogroupes est spécifique de tribu, avec des "polymorphismes privés" de tribu, ce qui implique que le processus de tribalisation soit survenu très tôt dans l'histoire des Amérindiens, suivi d'un relativement pauvre échange génétique intertribal.

L'étude de l'ADNmt sur des corps momifiés datant de 500 à 3000 ans de provenances variées (Illinois et Arizona - USA ; Chili) réalisée par différentes équipes retrouve ces quatre mêmes lignées d'ADNmt [7] confirmant ainsi les données du groupe de Wallace. Torroni et al. ont également étudié par les mêmes méthodes l'ADNmt de quatre cent onze individus provenant de dix populations aborigènes sibériennes vivant près du détroit de Behring. Les aborigènes sibériens ont trois des quatre haplogroupes (A, C et D) observés chez les Américains natifs alors que l'haplogroupe B n'est pas retrouvé dans ces populations [8]. La présence de l'haplogroupe $B$ chez des populations de l'Est asiatique et les Américains natifs et son absence chez les Sibériens proches du détroit de Behring font envisager que l'haplogroupe B pourrait provenir d'un événement migratoire distinct de ceux qui ont amené les groupes $\mathrm{A}$, $\mathrm{C}$ et $\mathrm{D}$ en Amérique.

Le scénario selon Torroni pourrait alors être le suivant : entre 17000 et
34000 années AM, quatre femmes (ou quatre groupes composés chacun de femmes génétiquement proches), porteuses des haplotypes ancestraux $\mathrm{A}, \mathrm{B}, \mathrm{C}$ et $\mathrm{D}$, pénétrèrent dans le Nouveau Monde venant d'Asie. La date avancée repose sur des calculs de variations des séquences, estimées à partir des sites de restriction des haplotypes et du taux consensus de l'évolution de l'ADNmt (2 à $4 \%$ par million d'années). La femme (ou le groupe de femmes) porteuse de l'haplotype ancestral A venait du SudEst de la Sibérie et migra plus tard que les autres en empruntant une voie différente, le long de la côte de la Sibérie par exemple, évitant ainsi les contacts avec les habitants de la toundra de la Sibérie de l'Est. C'est ce qui explique la plus faible variabilité génétique de l'haplogroupe $B$ par rapport à celle des haplogroupes $\mathrm{A}, \mathrm{C}$ et D. Ces groupes fondateurs ne trouvèrent personne pour limiter leur espace et leurs ressources. Ils s'étendirent donc rapidement dans tout le Nouveau Monde et se multiplièrent dans l'Amérique du Nord, l'Amérique centrale et l'Amérique du Sud. Puis, entre 12500 et 6000 années $\mathrm{AM}$, se produisit une deuxième migration d'une femme (ou d'un groupe de femmes) porteuse de l'haplotype ancestral A, donnant naissance à la population NaDene.

Mais tout le monde est loin d'être d'accord sur ce scénario. Tout d'abord, l'hypothèse de la route costale pour la population de l'haplogroupe B n'est pas totalement satisfaisante car il existe un gradient de fréquence de cet haplogroupe, avec une fréquence plus élevée dans le Sud. Une hypothèse alternative serait que ce groupe ait été formé par des voyageurs ayant traversé le Pacifique sans passer par le détroit de Behring, et correspondant au complexe culturel Lapita qui date d'à peu près 6000 ans (m/s $n^{\circ} 4$, vol. 10, p. 467) [9]. Ensuite, et surtout, Rick Ward, un généticien de l'université de l'Utah, et ses collaborateurs ont publié en 1991 un article où ils rapportent le séquençage de 360 nucléotides de l'anse $\mathrm{D}$ de l'ADNmt chez soixantetrois Amérindiens de la côte NordOuest appartenant à la tribu des Nuu-Chah-Nulth [10]. Ils avaient bien pu grouper la majorité des individus en quatre groupes qui, on le sait maintenant, correspondent aux quatre groupes de Torroni et Wallace. Mais, selon eux, la diversité entre les groupes est telle qu'elle n'a pu apparaître lors de l'entrée dans le Nouveau Monde. Ces quatre lignées ne sont pas nécessairement celles des quatre premières femmes américaines mais pourraient être quatre lignées survenues en Asie et qui ont commencé à muter en Asie. De plus, comme l'équipe de Graciela Bailliet en Argentine qui a étudié la diversité de l'ADNmt chez cent neuf Amérindiens d'Amérique du Sud appartenant à trois tribus (Mapuches, Huilliches et Atacamenos) [11], cette équipe a mis en évidence plusieurs autres haplotypes fondateurs potentiels en plus des quatre haplogroupes majeurs. Ansi, la migration dans le Nouveau Monde ne se serait pas accompagnée d'un sévère goulot d'étranglement génétique et, à la place de vagues bien individualisées, il y aurait eu un passage de l'Asie en "goutte à goutte " continu. En effet, d'un point de vue physique, l'homme anatomiquement moderne a pu entrer en Amérique du Nord via le détroit de Behring n'importe quand pendant les 100000 années AM. Il a été suggéré que la meilleure période pour traverser le détroit était 40000 à 30000 ans AM. Mais la glace d'hiver, avant 65000 ans AM et après 13500 ans AM, peut avoir permis aux populations de migrer librement en respectant les contraintes saisonnières [9].

\section{Conclusion - perspectives}

Incontestablement, le débat sur l'origine des premiers Américains reste ouvert.

Les éléments de travail dont nous disposons ne sont que le visage actuel du passé. Certaines populations primitives peuvent avoir disparu à la suite d'épidémies, de guerres ou de migrations forcées survenues durant la conquête de l'Amérique. Des sites archéologiques peuvent avoir été recouverts par l'avancée de la mer sur les côtes, détruits par l'agriculture moderne ou pillés [9]. La génétique moléculaire pourrait
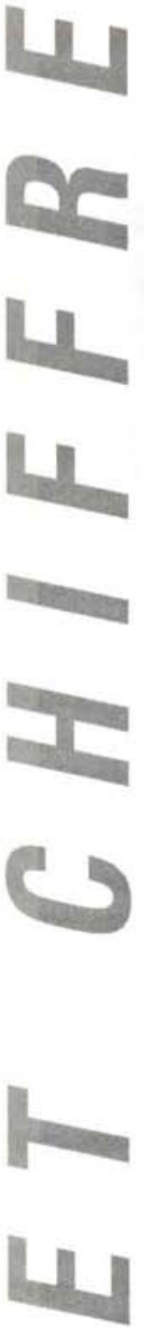

$\mathrm{m} / \mathrm{s} n^{\circ} 11$, vol. 10, novembre 94 
être utile en permettant d'étudier des marqueurs du chromosome $\mathrm{Y}$ afin de voir si les lignées d'ADN paternel présentent le même type de diversité que les lignées maternelles. Plus de gènes nucléaires et mitochondriaux peuvent également être analysés chez des Américains et des Asiatiques. Enfin, certains généticiens espèrent travailler sur plus d'anciens ADN comme ceux provenant de quatre-vingt-onze crânes datés de 8300 ans et trouvés dans un marais de Floride [7]

\section{RÉFÉRENCES}

1. Greenberg JA, Turner CG II, Zegura SL. The settlement of the Americas : a comparison of the linguistic, dental and genetic evidence. Cur Anthropol 1986 ; 27 : 477-98.

2. Cavalli-Sforza LL, Menozi P, Piazza A. Demic expansions and human evolution. Science $1993 ; 259: 639-46$.

3. Morell V. Confusion in earliest America. Science $1990 ; 248: 439-41$.

4. Marshall E. Clovis counterrevolution. Science $1990 ; 249: 738-41$.

5. Cann RL, Stoneking M, Wilson AC. Mitochondrial DNA and human evolution. Nature $1987 ; 325: 31-6$.

6. Torroni A, Schurr TG, Cabell MF, Brown MD, Neel JV, Larsen M, Smith DG, Vullo CM, Wallace DC. Asian affinities and continental radiation of the four founding native American mtDNAs. Am J Hum Genet 1993 ; 53 : 563-90.

7. Gibbons A. Geneticists trace the DNA trail of the first Americans. Science 1993 ; $259: 312-3$.

8. Torroni A, Sukernik SI, Schurr TG, Starikovskaya YB, Cabell MF, Crawford MH, Comuzzie AG, Wallace DC. mtDNA variation of aboriginal Siberians reveals distinct genetic affinities with native Americans. Am J Hum Genet 1993 ; 53 : 591-608.

9. Cann RL. mtDNA and native Americans : a Southern perspective. Am J Hum Genet $1994 ; 55: 7-11$.

10. Ward RH, Frazier BL, Dew-Jager K, Päảbo $S$. Extensive mitochondrial diversity within a single Amerindian tribe. Proc Natl Acad Sci USA $1991 ; 88: 8720-4$.

11. Baillet G, Rothhammer F, Carnese FR, Bravi CM, Bianchi NO. Founder mitochondrial haplotypes in Amerindian populations. Am J Hum Genet 1994 ; 54 : 27 - 